\title{
O Lúpus eritematoso sistêmico e seu processo de adoecimento: uma concepção feminina
}

\author{
A Systemic lupus erythematosus and its diseases process: a female conception
}

El lupus eritematoso sistémico y su proceso de enfermedad: una concepción femenina

\begin{abstract}
Patrícia dos Santos Lima ${ }^{1 *}$, Danyela Marina Bezerra Carvalho ${ }^{1}$, Joseana Martins Soares de Rodrigues Leitão ${ }^{1}$, Charllyton Luis Sena da Costa ${ }^{1}$, Heleny Noberto de Moura ${ }^{1}$, Lorrayne Monteiro Santos $^{2}$, Esdras de Sá Bezerra Neto ${ }^{1}$, Maria Vilmária de Oliveira Carvalho², Josefa Aliene de Sá Sousa ${ }^{1}$.
\end{abstract}

\section{RESUMO}

Objetivos: Identificar a concepção das portadoras de Lúpus Eritematoso Sistêmico (LES) desde o momento do diagnóstico ao decurso do tratamento. Métodos: $O$ delineamento deste estudo é transversal, descritivo, com a incorporação de um método quali-quantitativo, o qual foi submetido ao Comitê de Ética em Pesquisa (CEP). O Componente Especializado em Assistência Farmacêutica (CEAF) é o local de pesquisa, onde as portadoras cadastradas no sistema Hórus foram entrevistadas em grupos. Realizou-se também, a análise de dados documentais das pacientes registradas no sistema que se propuseram a participar do estudo. Resultados: A maioria das pacientes estão dentro dos perfis em que se encaixam as portadoras de lúpus, prevalecendo a faixa etária de 42 a 50 anos $(42,9 \%)$ e fora do período de menopausa $(57,1 \%)$, com diagnóstico a mais de cinco anos (57,1\%). Cerca de 57,1\% reclamam das manifestações clínicas, porém $42,9 \%$ não buscam novas alternativas de tratamento e apenas $14,3 \%$ frequentam grupos de apoio. Ao receber o diagnóstico $92,9 \%$ das mulheres reagiram de forma negativa, o que desencadeou problemas emocionais, como baixa autoestima e depressão. Atualmente, com o início do tratamento, $64,3 \%$ acreditam na cura para a doença. Conclusão: Através deste estudo foi possível identificar diversas concepções e elaborar um perfil epidemiológico aprofundado, onde estes dados servirão de base para futuras ações assistenciais, de forma ampla e efetiva, buscando sempre a melhoria na qualidade de vida das pacientes.

Palavras-chave: Assistência farmacêutica, Doenças autoimunes, Lúpus eritematoso sistêmico.

\begin{abstract}
Objectives: To identify the design of patients with systemic lupus erythematosus (SLE) from the time of diagnosis to the course of treatment. Methodology: The design of this study is transversal, descriptive, with the incorporation of a qualitative-quantitative method, with it was submitted to the Research Ethics Committee (CEP). The Specialized Component in Pharmaceutical Assistance (CEAF) is the research site, where the carriers registered in the Horus system were interviewed in groups. We also performed the analysis of documentary data of patients enrolled in the system who proposed to participate in the study. Results: The majority of the patients are within the profiles that fit the lupus carriers, with a prevalence of 42 to 50 years $(42.9 \%)$ and out of the menopause period $(57.1 \%)$, with a diagnosis of more than five years (57.1\%). About $57.1 \%$ complained about clinical manifestations, but $42.9 \%$ did not seek new treatment alternatives and only $14.3 \%$ attended support groups. On receiving the diagnosis $92.9 \%$ of the women reacted negatively, which triggered emotional problems, such as low self-esteem and depression. Currently, with the start of treatment, $64.3 \%$ believe in the cure for the disease. Conclusion: Through this study it was possible to identify several conceptions and to elaborate an in-depth epidemiological profile, where these data will serve as a basis for future care actions, in a broad and effective way, always seeking the improvement in patient's quality of life.
\end{abstract}

Keywords: Pharmaceutical care, Autoimmune diseases, Systemic lupus erythematosus.

${ }^{1}$ Centro Univ. Santo Agostinho-UNIFSA, Teresina-PI. * E-mail: patriciasantoslima2014@ hotmail.com.br

${ }^{2}$ Faculdade de Ensino Superior de Floriano - FAESF, Floriano - PI. 


\section{RESUMEN}

Objetivos: Identificar laconcepción de las portadoras de Lupus Eritematoso Sistémico (LES) desde el momento del diagnóstico al curso del tratamiento. Métodos: El delineamiento de este estudio es transversal, descriptivo, con la incorporación de un método cuali-cuantitativo, el cual fue sometido al Comité de Ética en Investigación (CEP). El Componente Especializado en Asistencia Farmacéutica (CEAF) es el lugar de investigación, donde las portadoras registradas en el sistema Horus fueron entrevistadas en grupos. Se realizó también, el análisis de datos documentales de las pacientes registradas en el sistema que se propusieron a participar del estudio. Resultados: La mayoría de las pacientes están dentro de los perfiles en que encajan las portadoras de lupus, prevaleciendo la franja de edad de 42 a 50 años (42,9\%) y fuera del período de menopausia (57,1\%), con diagnóstico a más de cinco años (57,1\%). Cerca del $57,1 \%$ se quejan de las manifestaciones clínicas, pero el $42,9 \%$ no buscan nuevas alternativas de tratamiento y apenas el 14,3\% frecuentan grupos de apoyo. Al recibir el diagnóstico el 92,9\% de las mujeres reaccionaron de forma negativa, lo que desencadenó problemas emocionales, como baja autoestima y depresión. Actualmente, con el inicio del tratamiento, el $64,3 \%$ cree en la cura para la enfermedad. Conclusión: A través de este estudio fue posible identificar diversas concepciones y elaborar un perfil epidemiológico en profundidad, donde estos datos servirán de base para futuras acciones asistenciales, de forma amplia y efectiva, buscando siempre la mejora en la calidad de vida de las pacientes.

Palabras clave: Asistencia farmacêutica, Enfermedades autoinmunes, Lupus eritematoso sistémico.

\section{INTRODUÇÃO}

O lúpus eritematoso sistêmico (LES) é uma doença inflamatória autoimune de natureza crônica que afeta diversos órgãos e sistemas do organismo. A doença atinge 30-50/10.000 pessoas em todo o mundo e incide mais frequentemente em mulheres jovens, na fase reprodutiva, acometendo 10 a 12 mulheres para cada homem (SOUSA et al., 2017). Segundo Brasil (2013), portaria GM/MS № 100, de 07 de fevereiro de 2013, que trata do Protocolo Clínico e Diretrizes Terapêuticas (PCDT) do lúpus eritematoso, no Brasil, estima-se uma incidência de LES em torno de 8,7 casos para cada 100.000 pessoas por ano, de acordo com um estudo epidemiológico realizado na região nordeste.

Conforme descreve Soares (2015), a etiologia do lúpus não está integralmente compreendida. Todavia, reconhece-se que existe uma interação dinâmica e complexa entre fatores genéticos, ambientais e hormonais, que predispõem a fenômenos imunológicos disruptivos incluindo disfunção de células dendríticas, células $\mathrm{B}$ e $\mathrm{T}$, produção de auto anticorpos e células $\mathrm{T}$ autorreativas. Dos fatores ambientais capazes de modular respostas do sistema imunitário salientam-se agentes infecciosos, químicos e outros poluentes e os fatores comportamentais, como o tabaco e a dieta. O stress psicossocial é dos principais fatores apresentados na literatura que implica na patogenia e precipitação de doenças autoimunes, dentre elas, o lúpus.

Segundo Hilbig et al. (2017), a principal característica relacionada a fisiopatologia do LES é a produção de auto anticorpos e imunocomplexos, com ativação do sistema complemento, afetando vários sistemas, sendo os sintomas gerais ou específicos, variando de acordo com a região acometida. As áreas mais afetadas são a pele, mucosas, serosas, sistema musculoesquelético, rins, sistema nervoso central (SNC), pulmão, coração, trato digestivo e sangue.

As formas leves da doença são assim descritas: fadiga, sintomas musculoesqueléticos, lesões cutâneas, pleuropericardite e pequenas alterações sanguíneas que interferem com a capacidade funcional e afetam a qualidade de vida. Já as formas graves se apresentam como, ou evoluem para, diversos tipos de glomerulonefrites, envolvem os vários componentes do sistema nervoso central (SNC), comprometem as funções cardiopulmonares, destroem rapidamente as séries sanguíneas periféricas ou alteram a função medular globalmente, levando a danos tissulares permanentes, às vezes irreversíveis, imputados como fatores de mau prognóstico (SANTIAGO et al., 2014). Pode ocorrer, também, com menor frequência, necrose asséptica, principalmente, do quadril, em função de vasculite e/ou da corticoterapia. Observam-se ainda alterações dermatológicas, como fotossensibilidade, rash malar (especialmente a "asa de borboleta"), 
lesão discóide, bem como alopecia difusa temporária e ulcerações e vasculite da mucosa oral (RIBEIRODO-VALLE, 2015).

Conforme afirma Brasil (2013), entre os medicamentos mais utilizados podem ser citados os antimaláricos (como a Cloroquina e Hidroxicloroquina) e glicocorticoides (como a Prednisona, Betametasona e Dexametasona), independentemente do órgão ou sistema afetado pela doença. O SUS ainda preconiza como possíveis alternativas para o tratamento o uso de Azatioprina, Ciclosporina, Ciclofosfamida, Danazol, Metotrexato e Talidomida.

Segundo Da Costa e Coimbra (2018), a terapia medicamentosa deve ser feita individualmente, segundo as necessidades do paciente e da gravidade da doença (agudo e/ou crônico), observando aspectos que vão desde a adesão ao tratamento até apoio familiar e dos diversos profissionais de saúde; necessitando um acompanhamento psicológico, a prática de atividades físicas regulares, uma dieta balanceada, sendo importante evitar fontes de raios ultravioletas e exposição ao sol, além de utilizar protetores e bloqueados solares.

O maior prejuízo se deve às alterações físicas e emocionais ocasionadas pelo processo patológico, especialmente nos períodos de exacerbação da doença. Nesse sentido, a mulher com LES vivência uma brusca mudança nos seus hábitos de vida. A rotina, as ocupações e tarefas que eram executadas até então podem ser alteradas, forçando a paciente a adaptar-se a novas exigências e a superar dificuldades e obstáculos que até o momento não eram vivenciados. E, embora a sobrevida dos pacientes com lúpus tenha aumentado nos últimos 50 anos, a qualidade de vida continua a apresentar baixos índices. Assim, o efeito das alterações causadas pelo processo patológico e pela terapêutica no curso clínico dessa doença demanda medidas que favoreçam a qualidade de vida como ferramenta essencial de satisfação para pacientes e profissionais de saúde (DA SILVA et al., 2016).

No Brasil são raros os estudos que chamam atenção para os transtornos depressivos em pacientes com LES. Investigar esta relação pode contribuir para intervenções terapêuticas mais efetivas. Além disso, os estudos deixam dúvidas se os estados depressivos poderiam contribuir para a não adesão ao tratamento (NEDER et al., 2015).

Diante disso, o presente estudo teve por objetivo identificar as concepções das portadoras de Lúpus Eritematoso Sistêmico, assistidas pela equipe do Componente especializado em Assistência Farmacêutica (CEAF), desde o momento do diagnóstico ao decurso do tratamento, para averiguar quais eram as principais dificuldades e progressos das pacientes com o decorrer do tempo. Esperava-se também traçar um perfil epidemiológico destas mulheres, visando intervenções futuras na qualidade de vida das mesmas.

\section{MÉTODOS}

O delineamento deste estudo constituiu-se de forma transversal, descritiva, com a incorporação de um método quali-quantitativo, onde o mesmo foi submetido ao Comitê de Ética em Pesquisa (№ CAAE: 88674518.2.0000.5602), com a finalidade de avaliar os aspectos éticos, para garantir os direitos, a segurança e o bem-estar dos sujeitos envolvidos neste projeto. O Componente Especializado em Assistência Farmacêutica (CEAF) é o local de pesquisa, localizado na cidade de Teresina, capital do estado do Piauí, onde são assistidas cerca de 79 patologias, possuindo um fluxo diário de aproximadamente 550 pacientes e mensal de 4500 .

O CEAF utiliza o sistema Hórus, que se trata de um sistema web onde são cadastrados os dados dos pacientes preenchidos pelos gestores, ficando armazenadas todas as informações desde exames até como se deve proceder a dispensação para o paciente. A amostra foi composta apenas por pacientes do sexo feminino, de faixa etária variada, que estavam cadastradas no sistema e utilizavam o Componente Especializado em tratamento para o LES no estado do Piauí, onde estas preencheram os critérios de diagnóstico e classificação de lúpus de acordo com PCDT de 2013 e aceitaram as condições propostas no Termo de Consentimento Livre e Esclarecido (TCLE). 
Foram excluídas da pesquisa as portadoras que não utilizavam os medicamentos preconizados no PCDT, disponibilizados no CEAF; as pacientes com diagnóstico e classificação que divergem do PCDT de Lúpus Eritematoso Sistêmico do Ministério da Saúde e as mulheres que não aceitaram as condições propostas dentro do TCLE. Desta maneira, foram entrevistadas 14 pacientes que estavam dentro dos critérios de inclusão. Também foi realizada a análise de dados documentais das pacientes registradas no sistema que se propuseram a participar do estudo. Todos os dados coletados foram analisados pelo programa Statistical Package for the Social Sciences (SPSS) e organizados em gráficos e tabelas para facilitar a compreensão dos leitores.

\section{RESULTADOS}

Foram entrevistadas 14 pacientes que estavam dentro dos critérios de inclusão. De acordo com a Tabela 1, a maioria das pacientes estão dentro dos perfis em que se encaixam as portadoras de lúpus, na faixa etária de 42 a 50 anos $(42,9 \%)$ e fora do período de menopausa $(57,1 \%)$.

Tabela 1. Características gerais das pacientes com Lúpus Eritematoso Sistêmico (Teresina - PI), Brasil, 2018. ( $n=14)$.

\begin{tabular}{|c|c|c|}
\hline CARACTERÍSTICAS & $\mathbf{n}$ & $\%$ \\
\hline \multicolumn{3}{|l|}{ FAIXA ETÁRIA } \\
\hline 18 a 25 anos & 2 & $14,3 \%$ \\
\hline 26 a 33 anos & 0 & - \\
\hline 34 a 41 anos & 2 & $14,3 \%$ \\
\hline 42 a 50 anos & 6 & $42,9 \%$ \\
\hline Acima de 50 & 4 & $28,6 \%$ \\
\hline \multicolumn{3}{|l|}{ ESTADO CIVIL } \\
\hline Solteira & 3 & $21,4 \%$ \\
\hline Casada & 9 & $64,3 \%$ \\
\hline Viúva & 0 & - \\
\hline Divorciada & 2 & $14,3 \%$ \\
\hline \multicolumn{3}{|l|}{ OCUPAÇÃO } \\
\hline Desempregada & 5 & $35,7 \%$ \\
\hline Empregada & 7 & $50 \%$ \\
\hline Aposentada & 1 & $7,1 \%$ \\
\hline Afastada & 1 & $7,1 \%$ \\
\hline \multicolumn{3}{|l|}{ FILHOS } \\
\hline Antes do diagnóstico & 6 & $42,9 \%$ \\
\hline Depois do diagnóstico & 2 & $14,3 \%$ \\
\hline Antes e depois do diagnóstico & 2 & $14,3 \%$ \\
\hline Nenhum & 4 & $28,6 \%$ \\
\hline \multicolumn{3}{|l|}{ MENOPAUSA } \\
\hline Sim & 6 & $42,9 \%$ \\
\hline Não & 8 & $57,1 \%$ \\
\hline \multicolumn{3}{|l|}{ ESTERECTOMIA } \\
\hline Sim & 2 & $14,3 \%$ \\
\hline Não & 12 & $85,7 \%$ \\
\hline
\end{tabular}

Fonte: Dados da pesquisa, 2018.

O esquema terapêutico (Figura 1) teve grande variação, prevalecendo o esquema 9 com 28,6\%. Os esquemas 1 (apenas Azatioprina) e 7 (apenas Corticoides) foram descartados pelas pacientes. Todas as 
pacientes afirmam ter recebido apoio dos profissionais de saúde e que tiveram melhora no quadro clínico com o início do tratamento.

Figura 1. Esquemas terapêuticos (Teresina - PI), Brasil, 2018. $(n=14)$.

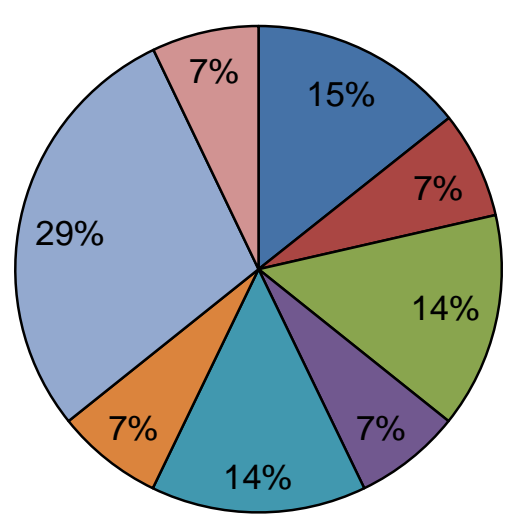
口Esquema 2: Hidroxicloroquina
口Esquema 3: Metotrexato
口Esquema 4: Azatioprina + Corticoides
口Esquema 5: Azatioprina + Hidroxicloroquina
口Esquema 6: Metotrexato + Hidroxicloroquina
口Esquema 8: Micofenolato de Mofetila
口Esquema 9: Azatioprina + Hidroxicloroquina
+ Corticoides

Fonte: Dados da pesquisa, 2018.

De acordo com as entrevistas realizadas, nenhuma paciente faz uso de álcool ou cigarro. Na Tabela 2 , cerca de $64,3 \%$ das mulheres realizam atividades físicas e $100 \%$ declararam que a doença não prejudicou as atividades de lazer e que praticam qualquer atividade que estejam ao seu alcance. Em relação ao uso de protetor solar $92,9 \%$ afirmaram utilizar protetor diariamente, além de não se expor ao sol. Com relação ao sono $50 \%$ sofrem de insônia e $85,7 \%$ utilizam outros medicamentos além do esquema terapêutico para tratamento do LES, dentre eles, medicamentos como calmantes e antidepressivos para dormir.

Em relação ao histórico e diagnóstico das portadoras de LES ( $\underline{\text { Tabela } 3}$ ), foram diagnosticadas a mais de cinco anos atrás $(57,1 \%$ e $92,9 \%$ das mulheres reagiram de forma negativa ao receber o diagnóstico, o que desencadeou problemas emocionais, como baixa autoestima e depressão.

Tabela 2. Hábitos de vida das pacientes entrevistadas (Teresina - PI), Brasil, 2018. ( $n=14)$.

\begin{tabular}{lcc}
\hline \multicolumn{1}{c}{ Hábitos } & $\mathbf{n}$ & $\%$ \\
\hline Atividades físicas & & \\
$\quad$ Diariamente & 9 & $64,3 \%$ \\
$\quad$ Esporadicamente & 1 & $7,1 \%$ \\
$\quad$ Não & 4 & $28,6 \%$ \\
\hline Atividade de lazer & 14 & \\
$\quad$ Sim & 0 & $100 \%$ \\
$\quad$ Não & & - \\
\hline Uso de protetor solar & 13 & \\
$\quad$ Sim & 1 & $92,9 \%$ \\
$\quad$ Não & & $7,1 \%$ \\
\hline Qualidade do sono & 0 & - \\
$\quad$ Excessivo & 7 & $50 \%$ \\
$\quad$ Regular & 7 & $50 \%$ \\
$\quad$ Insônia & & \\
\hline Uso de outros medicamentos & 12 & $85,7 \%$ \\
Sim & 2 & $14,3 \%$ \\
$\quad$ Não & &
\end{tabular}

Fonte: Dados da pesquisa, 2018. 
Tabela 3. Histórico e diagnóstico das portadoras de Lúpus (Teresina - PI), Brasil, 2018. (n=14).

\begin{tabular}{lcc}
\hline \multicolumn{1}{c}{ Histórico } & $\mathbf{N}$ & $\%$ \\
\hline Doenças na família & & \\
Doenças autoimunes & 4 & $28,6 \%$ \\
Doenças reumatoides & 4 & $28,6 \%$ \\
$\quad$ Depressão & 1 & $7,1 \%$ \\
Outras & 0 & - \\
$\quad$ Todas & 2 & $14,3 \%$ \\
$\quad$ Nenhuma & 3 & $21,4 \%$ \\
\hline Doenças anteriores & & \\
Sim & 8 & $57,1 \%$ \\
Não & 6 & $42,9 \%$ \\
\hline Diagnóstico & & \\
0-11 meses atrás & 2 & $14,3 \%$ \\
1-2 anos atrás & 3 & $21,4 \%$ \\
3-5 anos atrás & 1 & $7,1 \%$ \\
Mais de 5 anos & 8 & $57,1 \%$ \\
\hline Recorreu a vários profissionais & & \\
Sim & 13 & $92,9 \%$ \\
Não & 1 & $7,1 \%$ \\
\hline Sentimento ao receber o diagnóstico & & \\
Reação negativa & 13 & $92,9 \%$ \\
Nenhuma reação negativa & 1 & $7,1 \%$ \\
\hline Apoio familiar & & \\
Medo, desespero & 0 & - \\
Preconceito & 0 & - \\
Apoio e compreensão & 13 & $92,9 \%$ \\
Não sabiam o que significava & 1 & $7,1 \%$ \\
\hline Fon & &
\end{tabular}

Fonte: dados da pesquisa, 2018.

Se tratando da situação atual e do prognóstico da doença (Tabela 4), cerca de 57,1\% reclamam das manifestações clínicas, onde apenas $14,3 \%$ frequentam grupos de apoio e $64,3 \%$ acreditam na cura para a doença.

\section{DISCUSSÃO}

O Lúpus eritematoso sistêmico é uma doença autoimune sistêmica que afeta diversos órgãos e sistemas do organismo, com incidência mais frequente em mulheres jovens, na fase reprodutiva e ocasionando limitações significativas além dos danos emocionais para as pacientes. $O$ objetivo principal deste estudo era identificar a concepção das portadoras de LES desde o momento do diagnóstico ao decurso do tratamento, onde os dados obtidos foram comparados com a literatura.

Em um estudo transversal entre 39 mulheres com diagnóstico confirmado de LES, as quais eram atendidas em clínica de reumatologia e ambulatório de hospital universitário localizados em Maringá Paraná, a grande maioria eram casadas (56,4\%), com faixa de idade prevalente de 37 a 60 anos (35,9\%), seguida de 18 a 24 anos (30,8\%) (DA SILVA e AMADEI, 2016). Assim, estes resultados ratificam os achados obtidos nesta pesquisa, conforme o que está descrito na Tab. 1, a maioria das pacientes estão na faixa etária de 42 a 50 anos $(42,9 \%)$, casadas $(64,3 \%)$ e ainda não estão no período de menopausa (57,1\%). Analisando ainda o diagnóstico (Tab. 3), cerca de $57,1 \%$ das mulheres foram diagnosticadas há mais de cinco anos atrás, o que confirma que o lúpus afeta mais comumente mulheres jovens em idade reprodutiva, em uma proporção de nove a dez para cada homem (CEZARINO et al., 2017). 
Tabela 4. Realidade e prognóstico das pacientes com Lúpus Eritematoso Sistêmico (Teresina $-\mathrm{Pl}$ ), Brasil, 2018. $(\mathrm{n}=14)$.

\begin{tabular}{|c|c|c|}
\hline DADOS & $\mathbf{n}$ & $\%$ \\
\hline \multicolumn{3}{|l|}{ QUEIXA ATUAL } \\
\hline Manifestações clínicas & 8 & $57,1 \%$ \\
\hline Preconceito & 0 & - \\
\hline Falta de medicamentos & 1 & $7,1 \%$ \\
\hline O uso contínuo de medicamentos & 3 & $21,4 \%$ \\
\hline Outros & 1 & $7,1 \%$ \\
\hline Todos & 0 & - \\
\hline Nenhuma queixa atual & 1 & $7,1 \%$ \\
\hline \multicolumn{3}{|l|}{ GRUPOS DE APOIO } \\
\hline Sim & 2 & $14,3 \%$ \\
\hline Não conhece nenhum & 10 & $71,4 \%$ \\
\hline Não conhece, mas tem interesse & 1 & $7,1 \%$ \\
\hline Não tem interesse & 1 & $7,1 \%$ \\
\hline \multicolumn{3}{|l|}{ CURA PARA O LÚPUS } \\
\hline Sim & 9 & $64,3 \%$ \\
\hline Não & 5 & $35,7 \%$ \\
\hline \multicolumn{3}{|c|}{ BUSCA NOVAS POSSIBILIDADES DE TRATAMENTO } \\
\hline Sim & 8 & $57,1 \%$ \\
\hline Não & 6 & $42,9 \%$ \\
\hline \multicolumn{3}{|c|}{ COSTUMA CONVERSAR SOBRE A DOENÇA } \\
\hline Sim & 9 & $64,3 \%$ \\
\hline Não & 5 & $35,7 \%$ \\
\hline
\end{tabular}

Fonte: Dados da pesquisa, 2018.

Em relação ao tratamento, segundo Freire et al. (2011), o controle da atividade da doença é realizado principalmente pelo uso de corticoides e imunossupressores, fármacos que possuem vários efeitos colaterais como hipertensão arterial, diabetes, osteoporose e neoplasias, entre outros. Por se tratar de uma doença crônica incurável, o objetivo do tratamento baseia-se na supressão da atividade da doença, que é reversível, como também na prevenção contra o surgimento de danos orgânicos causados pela doença e de efeitos colaterais secundários aos fármacos utilizados, além do controle de comorbidades associadas. Examinando a Fig. 1, o esquema terapêutico que prevaleceu foi o uso de Azatioprina, combinada com Hidroxicloroquina e Corticoides $(28,6 \%)$, pois a maioria das portadoras apresentavam sintomas exacerbados, sendo necessário o uso de medicamentos combinados. Na Tab. 2, observando os hábitos de vida percebe-se que $85,7 \%$ das entrevistadas fazem uso de outros medicamentos, como os antihipertensivos e antidepressivos e 50\% das mesmas sofrem de insônia, o que as levam a usar medicações para melhorar o sono.

Como afirma Dallarmi et. al. (2015), o diagnóstico do lúpus é perturbador e gera alterações psicológicas que contribuem para diminuição na qualidade de vida das pacientes. As repercussões psicológicas (sentimentos negativos) vivenciadas pelas pacientes portadoras de LES são sempre esperadas, visto que essa é uma doença que tem a dor como sintoma clássico e evolução crônica, exigindo muito dos seus 
portadores (SILVA et al., 2012). Como descrito na Tab. 3, a maior parte das mulheres reagiram de forma negativa ao descobrir a patologia, sendo o obstáculo principal a dificuldade de se chegar a um diagnóstico, necessitando recorrer a vários profissionais de saúde. Alguns pacientes só detectaram o lúpus pelo fato de terem casos de doenças autoimunes e reumatoides na família.

De acordo com Ferreira e Leão (2017), a presença do lúpus traz inúmeras modificações em várias dimensões, corporais, sociais, afetivas, nas quais, a vida que já havia algumas frustrações desde a mais tenra idade, é agredida em proporções muito maiores. Neste estudo, cerca de $28,6 \%$ das mulheres relataram caso de depressão anterior ao diagnóstico, seguidos de perda familiar $(28,6 \%)$ e separação $(7,1 \%)$, e em alguns casos tiveram mais de um fator combinado como a perda familiar seguida de profunda depressão (14,3\%).

Como relata De Alcântara et al. (2011), após o tempo decorrido do diagnóstico, é chegado o momento da compreensão do mesmo e essa etapa é relatada com dificuldade e tristeza, denotando os sentimentos de medo, angústia e depressão. Aspectos bem característicos do impacto relacionado à tomada de consciência do fato de ser portadora de uma doença que é incurável e exige tratamento contínuo e hospitalização, impõe limites ao indivíduo e requer constante adaptação. De acordo com a Tab. 4, as entrevistadas relataram que as manifestações clínicas $(57,1 \%)$, seguidas do uso contínuo dos medicamentos $(21,4 \%)$ são as principais queixas atuais em relação ao lúpus. Apesar de todas as portadoras responderem que o tratamento trouxe melhorias significativas, os sintomas da doença não cessaram por completo e o fato de usar as medicações continuamente é um incômodo para as mesmas, que sentem dificuldades em aderir a terapêutica e desejam por diversas vezes abandonar o tratamento. Conforme o exposto na Tab. 2, cerca de $92,1 \%$ das mulheres realizavam o uso do protetor solar e no entanto todas elas reclamaram por usá-lo continuamente e por não poder se expor ao sol, além dos gastos para adquirir este produto, não disponibilizado gratuitamente. Apesar de todas as queixas e dificuldades enfrentadas, observando os hábitos destas mulheres (Tab. 2), todas praticam atividades de lazer e boa parcela realizavam atividade física recomendada pelo médico. Algumas ainda relataram que, toda e qualquer atividade que estejam ao seu alcance serão realizadas, pois acreditam que a patologia não pode ser mais forte que a sua vontade de viver.

Resende et al. (2016), realizou uma pesquisa com 31 adolescentes (11-21 anos) com lúpus e 19 cuidadores (32-66 anos) e em geral, o tratamento foi visto de forma positiva (melhoria, esperança, ajuda), com participação ativa do paciente (conhecimento, obediência, horário, remédio). Os participantes com nível de instrução mais alto trouxeram, em comum, a valorização de a doença ser tratável, a percepção do resultado do tratamento e sentimentos de esperança e afeto nesse enfrentamento. Os dados encontrados na pesquisa corroboram com os achados em literatura, observando a Tab. 4, apenas $14,3 \%$ frequentam grupos de apoio e $64,3 \%$ acreditam na cura para a doença, sendo que grande maioria das mulheres que acreditam na cura devido ao seu nível de instrução e 57,1\% buscam novas possibilidades de tratamento. Um dado alarmante é o fato de que somente 14,3\% das entrevistadas frequentam grupo de apoio e 35,7\% das mulheres ainda sentem vergonha em conversar sobre a doença. Portanto, essas pacientes necessitam de acompanhamento psicológico e de orientações por parte dos diversos profissionais da saúde e o SUS também deve garantir esse acesso, para que haja um acompanhamento integral da saúde.

\section{CONCLUSÃO}

Através deste estudo foi possível identificar diversas concepções das portadoras de LES, reconhecendo os principais problemas que afetavam as pacientes. Também foi possível elaborar um perfil epidemiológico aprofundado, onde estes dados servirão de base para futuras intervenções assistenciais de forma ampla e efetiva, buscando sempre a melhoria na qualidade de vida destas pacientes. As portadoras de lúpus enfrentam diversas dificuldades em seu dia-a-dia. Portanto, é essencial acompanhamento psicológico e de orientações multiprofissional, de forma gratuita pelo Sistema Único de Saúde (SUS), trazendo assim novas informações e apoio a estas mulheres, visando o equilíbrio entre o físico e o emocional, essencial aos pacientes lúpicos. 


\section{REFERÊNCIAS}

1. BRASIL. Ministério da Saúde. Portaria nํ 100, de 07 de fevereiro de 2013. Aprova o Protocolo Clínico e Diretrizes Terapêuticas do Lúpus Eritematoso Sistêmico. Diário Oficial da União, Brasília, DF, 08 fev. 2013, seção 1, p. 67.

2. CEZARINO RS, CARDOSO JR, RODRIGUES KN et al. Chronic low back pain in patients with systemic lupus erythematosus: prevalence and predictors of back muscle strength and its correlation with disability. Revista brasileira de reumatologia, 57(5): 438-444. 2017.

3. DA COSTA LM, COIMBRA CCBE. Lúpus eritematoso sistêmico: incidência e tratamento em mulheres. Revista Uningá Review, 20(1). 2018.

4. DALLARMI A, HEIMOSKI FE, JORGE GM et al. Distúrbios de humor em pacientes com lúpus eritematoso sistêmico. Associação médica do Paraná, 73(2): 17. 2015.

5. DA SILVA EB, DE OLIVEIRA LIMA RB, SILVA JS et al. The systemic lúpus erythematosus and women's selfimage carrier. Revista de Enfermagem da UFPI, 5(1): 67-72. 2016.

6. DA SILVA JP, AMADEI, JL. Influência da atenção médica na qualidade de vida (WHOQOL-100) de mulheres com lúpus eritematoso sistêmico. Revista Brasileira de Reumatologia, 56(3): 198-205. 2016.

7. DE ALCÂNTARA GC, DE AGUIAR, CCM, MONTEIRO KCC. A vivência de mulheres com lúpus eritematoso sistêmico. Psicologia, (2) 1. 2011.

8. FREIRE EAM, SOUTO LM, CICONELLI RM. Medidas de avaliação em lúpus eritematoso sistêmico - Artigo de revisão bibliográfica. Revista Brasileira de Reumatologia, 2011.

9. HILBIG C, MARTINEZ JV, MARTINEZ JE et al. Necrose de medula óssea em paciente portadora de lúpus eritematoso sistêmico. Revista da Faculdade de Ciências Médicas de Sorocaba, 18 (4): 187-192. 2017.

10. NEDER PRB, FERREIRA EAP, CARNEIRO JRM. Relação entre Ansiedade, depressão e adesão ao tratamento em pacientes com Lúpus. Revista Paraense de Medicina, 29 (2): 7-15. 2015.

11. RESENDE OLC, BARBOSA MTS, SIMÕES, BFT et al. A representação do adoecer em adolescentes com lúpus eritematoso sistêmico. Revista Brasileira de Reumatologia, 56(5): 398-405. 2016.

12. RIBEIRO-DO-VALLE CC. Lúpus eritematoso sistêmico e infecção do trato urinário: agentes etiológicos, perfil de susceptibilidade, tratamento e seu resultado. Dissertação (Mestrado em Ciências na área de concentração em Clínica Médica) - Faculdade de Ciências Médicas. Universidade Estadual de Campinas, Campinas, 2015, 42 p.

13. SANTIAGO MPB, DANTAS NCB, CARVALHO SBF et al. Atividade, Gravidade e Prognóstico de pacientes com Lúpus Eritematoso Sistêmico após prima internação-Estudo de Série de Casos. Journal of Health \& Biological Sciences, 2 (2): 65-73. 2014.

14. SILVA ACS, AMORIM EC, SILVA GG et al. Qualidade de vida de pacientes com lúpus eritematoso sistêmico: estudo preliminar comparativo. Revista Brasileira Clínica Médica, 10 (5): 390-392. 2012.

15. SOARES DMM. Influência do stress psicossocial no Lúpus eritematoso sistêmico - Artigo de revisão bibliográfica. 2015.

16. SOUSA JR, ROSA EPC, NUNES IFDOC et al. Efeito da suplementação com vitamina D em pacientes com lúpus eritematoso sistêmico: uma revisão sistemática. Revista Brasileira de Reumatologia, 2017. 